\title{
Fault Diagnosis of Transmission Line using Feed Forward Neural Network
}

\author{
Aditya Biswas, D. Malathi
}

\begin{abstract}
The implementation of neural network for the fault diagnosis is to improve the dependability of the proposed scheme by providing a more accurate, faster diagnosis relaying scheme as compared with the conventional relaying schemes. It is important to improve the relaying schemes regarding the shortcoming of the system and increase the dependability of the system by using the proposed relaying scheme. It also provide more accurate, faster relaying scheme. It also gives selective schemes as compared to conventional system. The techniques for survey employed some methods for the collection of data which involved a literature review of journals, from review on books, newspaper, magazines as well as field work, additional data was collected from researchers who are working in this field. To achieve optimum result we have to improve following things: (i) Training time, (ii) Selection of training vector, (iii) Upgrading of trained neural nets and integration of technologies. AI with its promise of adaptive training and generalization deserves scope. As a result we obtain a system which is more reliable, more accurate, and faster, has more dependability as well as it will selective according to the proposed relaying scheme as compare to the conventional relaying scheme. This system helps us to reduce the shortcoming like major faults which we faced in the complex system of transmission lines which will helps in reducing human effort, saves cost for maintaining the transmission system.
\end{abstract}

Keywords: Transmission Line, Faults, Artificial Intelligence, Multilayer Feed Forward Neural Network, Backpropagation, Genetic Algorithm

\section{I.INTRODUCTION}

The implementation of neural network for the fault diagnosis is to improve the dependability of the proposed scheme by providing a more accurate, faster diagnosis relaying scheme as compared with the conventional relaying schemes. It is important to improve the relaying schemes regarding the shortcoming of the system and increase the dependability of the system by using the proposed relaying scheme. It also provide more accurate, faster relaying scheme. It also gives selective schemes as compared to conventional system.

\subsection{Transmission Line Faults}

Electrical Power System (ESP) consists of three major parts in which the former part is generator, the transmission in middle and later is distribution part. Transmission line (TL) is one of important part of EPS network. It is mainly divided into two parts which are overhead transmission line

Revised Manuscript Received on July 18, 2019.

Aditya Biswas, M.Tech Student, Department Of Computer Science and Engineering, S.R.M Institute Of Science And Technology, Kattankulathur Campus, Kancheepuram, Tamil Nadu, India.

(Email: adityabiswas1994@gmail.com)

D. Malathi, Proffessor, Department Of Computer Science and Engineering, S.R.M Institute Of Science And Technology, Kattankulathur Campus, Kancheepuram, Tamil India. (E-mail: malathi.d@ktr.srmuniv.ac.in) and underground transmission line. Overhead Transmission line is more like affected to the fault than the other two parts, because it is very long and has greater stretch. The Neural Network is suitable way for the determination of the fault on TL. This system will helps us to classify the fault types such as series fault (one or two line open faults) or even shunt fault like (Single Line to ground, Double line to ground, 3PH (3- $\Phi)$ Ground

$3 \mathrm{PH}-\mathrm{G}$ as well as Single line, Double line, 3- $\Phi$ fault).Faults are the most challenging part for the transmission line. So it is mainly divided into two different categories, Normal Faults and Abnormal Faults. Normal Faults are those faults which occur due external influences such as short circuit faults due to strong winds, heat etc. and the Abnormal Fault are those faults which occur due to internal influences which mainly consist of error in flow design.

\subsection{Artificial Intelligence and Artificial Neural Network} $(A N N)$

The Artificial intelligence is performing action using three different methods which are as follows: (i) Expert System, (ii) Fuzzy Logic, (iii) Artificial Neural Network (ANN)

Expert System, this is widely used method. This system consist involvement of both human and system. The structure of this system is having an interface from where the system receives input from the user. After getting the input the interface engine compares the value with knowledge database and its explanation, as well as it also compare data along with the previously acquired data. After concluding it prints the output.

The Fuzzy Logic, it is a system consists of multi-valued system. This system deals with the partial truth, the result which will range from complete truth to complete false. This system uses Boolean logic 0 and 1 for delivering the output Sometimes it is very difficult to translate the output value and get proper information. The working of the system has some closer resembles to the working of the brain. But this system runs on probabilistic result which sometimes makes the system inefficient to deliver proper decision and output.

Artificial Neural Network is a design is a neural system creating an action which is resemble to the actions of biological brain. It is divided into three layers, 1. Input Layer, 2. Hidden Layer, 3. Output Layer, where we give input in the form of commands, datasets, Line of code. At Input layer, node were the artificial neural use to receive 
input, which as similar to neuron receptors, hidden layer were the processing and evaluation of data in done, and the output end gives result or output, which act similar to neuro cell transmitter part. At hidden layer, the processing is done with iteration which is known as epoch. During each epoch the processing and evaluation of given input (values) were done. The hidden layer can be taken for nth number of layer. The ANN is system is performed with various processes such as Back-propagation, Feed-Forward method, FeedBackward method, using Genetic Algorithm.

\section{SURVEY METHODOLOGY}

\subsection{Planning}

The steps were taken for this survey paper:

Survey of literature: This process involves literature books, referring of various journals paper, technological magazines etc. to gather information regarding the Artificial Intelligence methods used in fault diagnosis of transmission line (time-span approximately10 years).

$$
\text { - }
$$

Analysis of data: Gathering of relevant data from the research papers, conference paper as well as various survey papers.
-

work.

Gathered information during the field

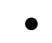

Comparison of data: All data is analyzed and gone through validity checking within this survey.

Conclusion: Achieving relevant data and apply it on the proposed system for obtaining the optimum result.The Survey method involves various questionnaire surveys, referring various journals, conference paper, and other survey papers. The following stages were used throughout the analysis: (i) Collecting all kind information and data; (ii) Categorizing the applications for the usage of data; (iii)Train the data for the quality data and case studies; and (iv) Refining the data.

\section{ARTIFICIAL INTELLIGENCE - OVERVIEW}

In this section, definition of terms artificial intelligence is there, together with a comparison of human and artificial intelligence. The artificial intelligence is performing action using three different methods which are as follows: (i) expert system, (ii) fuzzy logic, (iii) artificial neural network (ann).

Expert system is widely used method. This system consist involvement of both human and system. The structure of this system is having an interface from where the system receives input from the user. After getting the input the interface engine compares the value with knowledge database and its explanation, as well as it also compare data along with the previously acquired data. After concluding it prints the output.

The fuzzy logic is a system consists of multi-valued system. This system deals with the partial truth, the result which will range from complete truth to complete false. This system uses boolean logic 0 and 1 for delivering the output. Sometimes it is very difficult to translate the output value and get proper information. The working of the system has some closer resembles to the working of the brain. But this system runs on probabilistic result which sometimes makes the system inefficient to deliver proper decision and output.

Artificial neural network is a design is a neural system creating an action which is resemble to the actions of biological brain. It is divided into three layers, (i) input layer, (ii) hidden layer, (iii) output layer, where we give input in the form of commands, datasets, line of code. At input layer, node were the artificial neural use to receive input, which as similar to neuron receptors, hidden layer were the processing and evaluation of data in done, and the output end gives result or output, which act similar to neuro cell transmitter part. At hidden layer, the processing is done with iteration which is known as epoch. During each epoch the processing and evaluation of given input (values) were done. The hidden layer can be taken for $n^{\text {th }}$ number of layer The ann is system is performed with various processes such as back-propagation, feed-forward method, feed-backward method, using genetic algorithm.

\section{ARTIFICIAL INTELLIGENCE IN ELECTRICAL APPLICATION \& RESULTS}

In this section, the focus is on explaining of AI method used in electrical system: Artificial Neural Network (ANN) and other algorithms. Electrical Power System (ESP) consists of three major parts in which the former part is generator, the transmission in middle and later is distribution part. Transmission line (TL) is one of important part of EPS network. It is mainly divided into two parts which are overhead transmission line and underground transmission line. Overhead Transmission line is more like affected to the fault than the other two parts, because it is very long and has greater stretch. The Neural Network is suitable way for the determination of the fault on TL. This system will helps us to classify the fault types such as series fault (one or two line open faults) or even shunt fault like (Single Line to ground, Double line to ground, 3PH (3- $\Phi$ ) Ground 3PH-G as well as Single line, Double line, 3- $\Phi$ fault). The initial data is collected from various journals, and conference papers from different years which are as follows:

The authors J. Upendar et al. [1] have describe fault grouping must be precise to encourage speedy repair of the framework, enhance framework accessibility and lessen working expenses due to mal-task of transfer. Artificial Neural Systems (ANSs) can be a viable procedure to anticipate the faults, when it is given qualities of fault current and the comparing past choices as yields. This paper describes the utilization of Particle Swarm Optimization (PSO) for a compelling preparing of ANN and the use of wavelet changes for anticipating the sort of faults. Through wavelet investigation, deficiencies are decayed into a progression of wavelet segments, every one of which is a period area flag that covers a particular octave recurrence band. The parameters chose for fault order are the nitty gritty coefficients of all the stage current signs, estimated at the sending end of a transmission line. The data is then bolstered into ANN for ordering the deficiencies. Thusly the

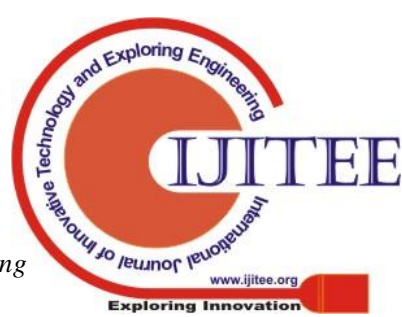


PSO-based perceptron approach can be utilized as an appealing and viable methodology for arrangement calculation of intensity framework flaws. The algorithms used by the authors are Back Propagation Neural Network (BPNN), Genetic Algorithm (GA), Discreet Wave Transform (DWT), Particle Swarm Optimization (PSO) algorithm. The optimization algorithm is exhibited to have the capacity to give model free estimation in deriving the yield from the input. Different case study has been examined including the variety of fault separation, beginning point and fault protections. Both PSO-based perceptron approach and BPNN strategy have been executed. The execution indicated exhibits that the proposed strategy gives a high exactness $(99.912 \%)$ in grouping of the power framework faults.

The authors T. S. Sidhu et al. [2] have proposed an exhaustive examination of an artificial neural system based fault bearing discriminator. Singular yields of neurons are researched. Another method dependent on backtracking look strategy is created for examining the multilayer feedforward neural systems. The method is connected to the fault heading discriminator's individual neurons. ANN as MLP backpropagation: Multilayered Feedforward Neural Network (MFNN). Three methodologies: Decomposition approach: There is legitimate thought of the inward structure of the neural system. Discovery approach: There is no thought of the inner design of the neural system. Electric methodology: This methodology is the cross breed of both decay and discovery approaches. Two different ways investigation should be possible i.e., Feedforward analysis and Reverse analysis. Less time taken for computational count. At the present, no techniques exist for analyzing the Feedforward neural system based transfers. The proposed strategy can be utilized for recognizing all input of info that will deliver a desired output for a prepared system. The use of the proposed method for analysis of the MFFN-based fault heading discriminator has featured a portion of the territories for which the conduct of the neural system might be eccentric. It is not necessarily the case that the ANNbased faults direction discriminator won't work, yet it needs additionally testing and assessment to ensure that it will work accurately for the distinguished cases.

The authors Ênio C. Segatto et al. [3] have present elective methodologies utilizing Artificial Neural Systems (ANNs) for the protection of power transformers. A total protection scheme was actualized. An ANN subroutine was utilized to separate inward faults from different circumstances, swapping the customary Fourier technique for harmonic restraint. Moreover, a daily schedule for remaking of soaked current signs dependent on intermittent ANNs is additionally proposed. The proposed techniques were widely tried and afterward contrasted with the customary differential security calculation, indicating promising outcomes. The utilization of the ANN apparatuses is another and essential stage in the differential transfer examination strategy for power transformer security. Elective methodologies utilizing ANNs for the insurance of intensity transformers. An ANN subroutine was utilized to segregate inside issues from different circumstances, incorporating into surge flows created amid the transformer activity. Moreover, a daily practice for reproduction of immersed current signs dependent on repetitive ANNs is likewise proposed. Customary calculation for differential protection of power transformers exercise harmonics self-control by Fourier change; First calculation proposed utilizing ANNs with MLP backpropagation strategy with saturated information; Second calculation proposed utilizing information reconstruction and pattern recognition. The precision of $98.65 \%$ and $97.80 \%$ for power transformers of $25 \mathrm{MVA}$ and 6.25 MVA, separately. The time reaction was $1 / 4$ of a cycle. The second methodology incorporated a daily schedule for saturation reconstruction. The best outcomes demonstrated the precision of $99.92 \%$ and $99.31 \%$ for power transformers of $25 \mathrm{MVA}$ and 6.25 MVA, individually. The time reaction was $1 / 2$ of a cycle. This cases tested by ANNs were then contrasted with the conventional differential protection calculation, which displayed inaccuracies.

The authors Monika Gupta et al. [4] have proposed the combination of Neural Systems and Genetic Algorithm can be utilized to frequency control framework recurrence. Neural system offers a great point of interest in learning, adjustment, adaptation to internal failure and parallelism. Genetic Algorithm is a parallel worldwide pursuit method that copies characteristic Genetic administrators. In the proposed calculation learning of weights of neural systems is finished utilizing Genetic Algorithm. The outcomes obtained by using simulation better execution of the proposed control structure when comparison done between traditional back propagation and least mean square calculation. A recurrence estimation procedure for a power framework neural system whose weights are got learnt of utilizing Genetic Algorithm is utilized for estimation of intensity framework. Practical conditions like frequency variation because of elements like sudden change in load, expansion of another unit to generation side and noises are viewed as frequency variation. The said system is prepared for 24 hours and estimation is improved the situation next 24 hours. Algorithms used by the authors' are Artificial Neural Network: Multilayer Feedforward neural system (MFFNN) with an error backpropagation (BP) calculation, Genetic Algorithm, Least Mean square Algorithm. Combination of neural systems and genetic algorithm can be utilized to appraise control framework frequency. Neural systems then again offer extraordinary focal points in learning, adjustment, adaptation to internal failure and parallelism. Genetic algorithm is a parallel worldwide pursuit system that imitates normal genetic operators. In the proposed algorithm learning of weights of neural systems is finished utilizing hereditary calculation. Times and distinctive faults types. Neural system whose weights are got the hang of utilizing genetic algorithm is utilized for estimation of power framework. Practical conditions like frequency differences because of elements like sudden change in load, expansion of another unit to generation side and irregular noise are additionally viewed as frequency. The said system is prepared for 24 hours and estimation is improved the situation next 24 hours.

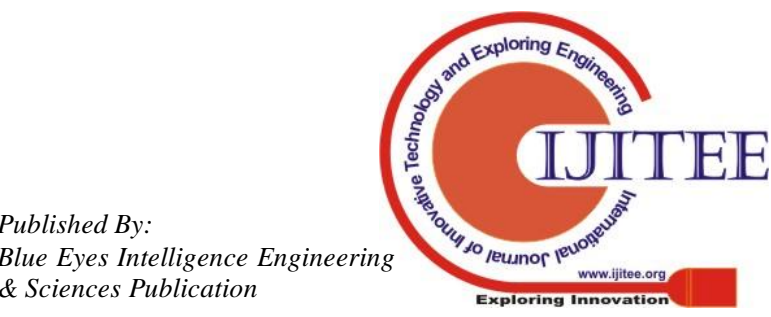


The authors J. Upendar et al. [5] have analyzed and concluded that Fault characterization must be precise to encourage speedy repair of the framework, enhance framework accessibility and diminish working expenses due to mal-operation of transfer. Artificial Neural Systems (ANS) can be a successful procedure to anticipate the fault, when it is furnished with qualities of fault flows and the relating past choices as output. This paper portrays the utilization of Particle Swarm Optimization (PSO) for a compelling preparing of ANN and the use of wavelet changes for foreseeing the sort of fault. Through wavelet examination, faults are disintegrated into a progression of wavelet parts, every one of which is a period area flag that covers a particular octave recurrence band. The parameters chose for fault classification are the point by point coefficients of all the stage current signs, estimated at the sending end of a transmission line. The data is then fed into ANN for characterizing the faults. Along these lines the PSO-based perceptron approach can be utilized as an alluring and successful methodology for arrangement calculation of intensity framework issues. The accompanying strategies and algorithms are for the most part utilized in this paper by the authors' are Back propagation Neural Network (BPNN), Genetic algorithm Swarm Optimization Algorithm(PSO Algorithm). The optimization calculation is exhibited to have the capacity to give display free estimation in concluding the output from the information. Different contextual investigations have been examined including the variety of fault separation, initiation edge and fault protections. Both PSO-based perceptron approach and BPNN strategy have been actualized. The execution indicated shows that the proposed method gives a high precision $(99.912 \%)$ in order of the power framework shortcomings.

The authors Mohammad Mohatram et al. [6] have proposed an artificial neural system in Electric Power Industry (EPI). A standout amongst the most exciting and conceivably financially savvy ongoing improvements in this field is expanding utilization of man-made consciousness methods viz. Artificial Neural systems (ANNs), Genetic calculation, fluffy rationale, and master frameworks. Techniques discussed by the authors' are Artificial Neural Networks (ANNs), Genetic algorithm (GA), Fuzzy logic (FL), and Expert systems (XPS). Fast regardless of the multifaceted nature of the issues. On-line processing and classification. Capability of giving with stochastic varieties of the planned working point with expanding information. Verifiable nonlinear demonstrating and separating of framework information consequently. Enormously parallel appropriated structure and capacity to learn. Assurance of different parameters like the quantity of hidden layers. Number of neurons in the concealed layer. Finding the ideal design of ANNs is an extremely tedious process. Rather than ordinary procedures or master frameworks, which endeavors to formalize learning and create incomplete quality framework models, ANN don't give a formal portrayal of the connection among info and output data, it is therefore fundamental to test the ANN execution by statistical tools. ANN experiences the prerequisite of master user in their structure and usage. They additionally (GA), Discreet Wavelet Transformation (DWT), Particle

experience the ill effects of an absence of the formal model hypothesis and scientific rigors as are presented to the specialists' profundity of learning in issue definition. ANNs depend on regular reproductions with the end goal to deliver preparing vectors and analysis the preparation vectors, particularly with loud information. There are some significant issues to be handled utilizing ANNs for power framework: preparing time, choice of preparing vector, overhauling of prepared neural nets and mix of advancements. ANN with its guarantee of versatile preparing and speculation merits scope for further examination.

The authors Mohamed M Ismail et al. [7] have proposed the propelled utilization of Artificial Neural Network was presented as of late in Protection of Transmission lines in Electric Power Systems. In this proposed research, the utilization of ANFIS and ANN for Distance Relay Protection for short and long Transmission line, under various stacking conditions, in Electrical Power frameworks will be presented and examined. Considering diverse stacking conditions, the recommended and proposed method manages fault identification, grouping, and area in short and long Transmission lines. The utilization of ANFIS and ANN for Distance Relay Protection for short and long Transmission line, under various stacking conditions, in Electrical Power. The proposed intelligent distance relay show successful execution for the three principle security assignments; fault discovery, fault grouping and fault area. For the fault area assignment, the most extreme error is around $8 \%$ for ANFIS locator and $10 \%$ for ANN identifier for various fault protections, diverse fault commencement times and distinctive fault composes. The testing methodology considers the arbitrariness of the shortcomings on T.Ls regarding the season of event, fault area, type and opposition and notwithstanding for the loading conditions.

The authors Ahmed Sabri Salman Altaie et al. [8] have proposed an objective of this exploration is to center and receive a quick, precise and solid fault recognition procedure and arrangement technique for the high voltage transmission line. In the first place, recognition of the fault is done utilizing another proposed procedure that consolidates three kinds of transfers. Second, the system disconnects the broken part in a quick time span. At last, characterizing the faults is completed by information recorded utilizing Digital Signal Processing (DSP) and Artificial Neural Network (ANN) in light of various ways The information preparing information of the account gadgets was inspected utilizing Digital Signal Processing (DSP). The information gathered from the recorders will be utilized to group the fault simply because the time isn't an essential factor as in fault recognizing and clearing. A wide range of shortcomings are researched for the fault order Three techniques are utilized (Phase Current examining, Phase Shift of the Phase Voltage testing and Phase Voltage inspecting) to assess the proficiency, exactness and the investigation the mean square mistake. Techniques used by the authors' are DSP sampling and ANN.

Published By: 

classification in transmission line has been made and summarized. The expansion of non-direct loads lately has a solid bearing on power quality because of the nearness of sounds in the power framework. mistaken estimation and failing of defensive transfers, along these lines decreasing the viability of transmission line assurance. With the point of enhancing the steadfastness of the insurance conspire under differing non-direct stacking condition, the authors' Ebha Koley et al. in [9] have introduced Support Vector Machine (SVM), Artificial Neural System (ANS) and Kalman channel based calculation utilizing voltage sounds for the assurance of three-stage transmission line. The accompanying strategies and algorithms are for the most part utilized in this paper are: Back propagation Neural Network(BPNN), Genetic algorithm(GA), Discreet Wavelet Transformation (DWT), Particle Swarm Optimization Algorithm(PSO Algo). The optimization calculation is shown to have the capacity to give display free estimations in concluding. The output from the information. Different contextual analyses have been contemplated including the variety of blame separation, initiation point and blame protections. Both PSO-based perceptron approach and BPNN strategy have been actualized. The execution indicated shows that the proposed method gives a high exactness $(99.912 \%)$ in arrangement of the power framework shortcomings.

The author Ahmad Abdullah [10] has proposed a new application of wavelet-based artificial neural networks (ANNs). The application uses high-frequency content of a subset of local currents of one end of a protected line to classify transients on the line protected and its adjacent lines. The scheme can classify transients-including faults - occurring on a protected line, categorize transients on adjacent lines, and pinpoint the line causing the transient event. Modal transformation is used to transform phase currents to modal quantities. Discrete wavelet transform (DWT) is used to extract high-frequency components of the two aerial modal currents. The contribution of the paper is as per the following. It has been demonstrated that flows alone can be utilized for transient mark of the occasion. Just two modes are important for characterization. Just a single eighth of a cycle of post event information is vital for classification. The methodology utilized by the author are, Decouple flows utilizing modal analysis. Apply the DWT to the airborne modes utilizing one-eighth of a cycle of post event flows. Stack the arrangement of coefficients of the two aerial methods of a specific level over one another to make a vector used to prepare ANN. A neural system of proper size is utilized for preparing. Preparing is finished utilizing transient situations. These situations incorporate shortcomings, line exchanging, and lightning. Highfrequency signs can be utilized for rapid power framework fault location through transient order recognizing the line causing the transient occasion. It has been demonstrated that flows alone can be utilized for transient mark of the occasion. Just two modes are essential for order. Just a single eighth of a cycle of post event information is fundamental for grouping. In spite of the fact that the outcomes have been indicated just to a particular tower, the creator has attempted diverse tower designs and affirmed
An elaborate analysis of survey on fault diagnosis and

that the calculation works for all tower arrangements considered. It ought to be certain that the present strategy falls flat if a lightning strike develops to a fault. The author has not expected any disappointment of protection in the simulation. The cutting of the signals related with protection breakdown can potentially mislead the plan proposed. Further investigation is expected to totally measure its impact on the proposed relaying scheme. It ought to be noted, be that as it may, that lightning strikes are being cleaved by the surge arrester yet at the same time being accurately recognized. Field approval is being performed and results will be shared once all examinations are finished.

\section{CONCLUSION}

In this paper, we have pointed out the various research works carried out by researcher for fault diagnosis, we can conclude that the Electrical Power System (ESP) is a very complex system. And the external transmission-lines is very likely to get affected from many external influences which result in arise of faults. Faults are the most challenging part for the transmission line. So it is mainly divided into two different categories, Normal Faults and Abnormal Faults. For this kind of complex system it is very difficult to locate and identify the fault. Applying ANN to this kind system will make the system faster, easy for the diagnosis and rectify the faults. It also increases reliability and the efficiency of the system.

\section{REFERENCES}

1. Tony B. Nguyen, Student Member, IEEE, and M. A. Pai, Fellow, IEEE, "Dynamic Security-Constrained Rescheduling of Power Systems Using Trajectory Sensitivities", IEEE Transactions on Power Systems, Vol. 18, No. 2, pp. 848-854, May 2003.

2. T. S. Sidhu, Fellow, IEEE, L. Mital, and Mohindar S. Sachdev, Life Fellow, IEEE, "A Comprehensive Analysis of an Artificial Neural-Network-Based Fault Direction Discriminator", IEEE Transactions On Power Delivery, Vol. 19, No. 3, Pp.1042-1048, July 2004

3. Ênio C. Segatto and Denis V. Coury, Member, IEEE, "A Differential Relay for Transformer Using Intelligent tools", IEEE Transactions On Power Systems, Vol. 21, No. 3, Pp.1154-1162, August 2006

4. Monika Gupta, Smriti Srivastava, and J.R.P Gupta, "Power System Frequency Estimation Using Neural Network and Genetic Algorithm", Joint International Conference on Power System Technology and IEEE Power India Conference, New Delhi, India, 12-15 Oct. 2008

5. J. Upendar, C.P. Gupta, G.K. Singh, G. Ramakrishna, "PSO and ANN-based fault classification for protective relaying”, IET Gener. Transm. Distrib., Vol. 4, Iss. 10 pp. 1197-1212, 2010

6. Mohammad Mohatram, Peeyush Tewari and Shahjahan, "Application of Artificial Neural Network in electric power industry", International Journal of Electrical Engineering, Volume 4, Number 2, pp.161-171, 2011

7. Mohamed M Ismail and M A. Moustafa Hassan, "Distance Relay Protection for Short and Long Transmission line", Intemational Conference on Modelling, Identification \& Control (ICMIC), Cairo, 
Egypt, 31stAug.- 2ndSept. 2013

8. Ahmed Sabri Salman Altaie and Johnson Asumadu, "Fault Detection and Classification for Compensating Network Using Combination Relay And ANN", IEEE International Conference on Electro/Information Technology (EIT), Dekalb, IL, USA, 21-23 May 2015

9. Ebha Koley, Sunil K. Shukla, Subhojit Ghosh, Dusmanta K. Mohanta, "Protection scheme fpr power transmission line based on SVM and ANN considering the presence of non-linear loads", IET Gener. Transm. Distrib. Vol. 11 Iss. 9, pp. 2333-2341, 2017

10. Ahmad Abdullah, Member, IEEE, "Ultrafast Transmission Line Fault Detection Using a DWT-Based ANN", IEEE Transactions On Industry Applications, Vol. 54, No. 2, March/April 2018 